\title{
Self-similar non-clustered planar graphs as models for complex networks
}

\section{Francesc Comellas}

Dep. de Matemàtica Aplicada IV, EPSC, Universitat Politècnica de Catalunya

Av. Canal Olímpic s/n, 08860 Castelldefels, Barcelona, Catalonia, Spain

E-mail: comellas@ma4.upc.edu

\section{Zhongzhi Zhang}

School of Computer Science, Fudan University, Shanghai 200433, China

Shanghai Key Lab of Intelligent Information Processing, Fudan University, Shanghai 200433, China

E-mail: zhangzz@fudan.edu.cn

\section{Lichao Chen}

School of Computer Science, Fudan University, Shanghai 200433, China

Shanghai Key Lab of Intelligent Information Processing, Fudan University, Shanghai 200433, China

E-mail: chenlichao@gmail.com 
Abstract. In this paper we introduce a family of planar, modular and self-similar graphs which have small-world and scale-free properties. The main parameters of this family are comparable to those of networks associated with complex systems, and therefore the graphs are of interest as mathematical models for these systems. As the clustering coefficient of the graphs is zero, this family is an explicit construction that does not match the usual characterization of hierarchical modular networks, namely that vertices have clustering values inversely proportional to their degrees.

PACS numbers: 02.10.Ox, 89.20.Ff, 89.75.Da, 89.75.-k 


\section{Introduction}

Research and studies performed in the last few years show that many networks associated with complex systems, like the World Wide Web, the Internet, telephone networks, transportation systems (including power and water distribution networks), social and biological networks, belong to a class of networks now known as small-world scale-free networks, see $[1,2]$ and references therein. These networks exhibit a small average distance and diameter (compared to a random network with the same number of nodes and links) and, in many cases, a strong local clustering (nodes have many mutual neighbors). Another important common characteristic is that the number of links attached to the nodes usually obeys a power-law distribution (is scale-free). Moreover, a degree hierarchy in these networks is sometimes related to the modularity of the system. By introducing a new measuring technique, it has been discovered that many real networks are self-similar and fractal [3,4]. More recently, a characterization of self-similarity versus fractality has been given in $[5,6]$.

Most of the network models considered are probabilistic, however in recent years a deterministic approach has proven useful to complement and enhance the probabilistic and simulation techniques. Deterministic models have the strong advantage that it is often possible to compute analytically many network properties, which may be compared with experimental data from real and simulated networks. Some deterministic models have been proposed which are very often based on iterative constructions like the so called $k$-trees [7]. A generic $k$-tree is a graph theoretical construction which starts at $t=0$ with a complete graph $K(d, 0)=K_{d}$ or $d$-clique. For any step $t \geq 1$, the $k$-tree $K(d, t)$ is constructed from $K(d, t-1)$ by selecting one or more existing $d$-cliques in $K(d, t-1)$ and adding, for each of them, a new vertex connected to all the vertices of the clique. Note that a $k$-tree is a graph which contains numerous cycles and hence it is not a tree in the strict sense.

Several modifications of this general construction have been considered. Networks are associated to the choice of the value $d$ and also to the way cliques are selected. For example, Dorogotsev, Golsev and Mendes [8] have considered $d=2$, and at each step all existing $K_{2}$ are used to add a new vertex. The dual network model was given by Jung, Kim and Kahng in [9]. The first construction can be generalized to any $d$, see [10]. For $d=3$, and related to the classical Apollonian packing of circles, Andrade et al. and Doye and Massen introduced and studied the so called Apollonian networks [11, 12]. These networks are also $k$-trees but new vertices are attached only to cliques which have never been selected in a former iteration. Two-dimensional Apollonian networks are simultaneously scale-free, small-world, Euclidean and space filling. Moreover, they are maximal planar graphs. The general case $d \geq 3$, high dimensional Apollonian networks, was introduced in [12] and has been further studied in [13].

A different technique produces graphs by duplication of certain substructures, see [14]. Here we propose a new family of graphs which generalizes these former methods by introducing at each iteration a more complex substructure than a single vertex. The 
result is a family of planar, modular, hierarchical and self-similar graphs, with smallworld scale-free characteristics and with clustering coefficient zero. We note that some important real life networks, for example the networks associated to electronic circuits or Internet [2], have these characteristics as they are modular, almost planar and with a reduced clustering coefficient and have small-world scale-free properties. Thus, these networks can be modeled by our construction. A related family of graphs based on triangles, and which therefore has a high clustering coefficient was introduced in [15].

\section{Hierarchical modular graphs}

Several authors classify as hierarchical graphs, graphs with a modular structure and a strong connectedness hierarchy of the vertices which produces a power-law degree distribution. Moreover, they consider that the most important signature of hierarchical modularity is given by a clustering distribution with respect to the degree according to $\mathcal{C}(k) \propto 1 / k$, see $[16,8]$. We recall that the clustering coefficient $c_{v}$ of a vertex $v$ with degree $\delta_{v}$ is the fraction of the number of present edges over the $\delta_{v}\left(\delta_{v}-1\right) / 2$ potential edges among the neighbors of $v$. The clustering of the graph is obtained averaging over all its vertices. In this section we define and analyze a family of hierarchical modular graphs, which are scale-free, planar and have clustering coefficient zero. They prove the existence of hierarchical graphs which do not have the above-mentioned relationship between the clustering coefficient and the degrees of the corresponding vertices.

Deterministic models for simple hierarchical networks have been published in $[17$, 18]. These models consider the recursive union of several basic structures (in many cases, complete graphs) by adding edges connecting them to a selected root vertex. These and other hierarchical graphs have been considered when modeling metabolic networks in $[19,20]$. Hierarchical modularity also appears in some models based on $k$-trees or clique-trees, where the graph is constructed by adding at each step one or more vertices and each is connected independently to a certain subgraph $[8,10,13]$. The introduction of the so-called hierarchical product of graphs in [21] allows a generalization and a rigorous study of some of these models.

In [3, 4], Song, Havlin and Makse relate the scale-free and the self-similarity properties as they verify that many self-similar graphs associated to real life complex systems have a fractal dimension and provide a connection between this dimension and the exponent of the degree power-law. However, a classical scale-free model, the preferential attachment by Barabási-Albert [1], which many authors consider a paradigm for these networks, has a null fractal dimension. This is not a paradox as the BarabásiAlbert model lacks modularity because its generation process is based on the individual introduction of vertices. Moreover, a condition for self-similarity, which is independent from fractality, has been provided recently in $[5,6]$.

In the next subsection we give details of our construction which is also based on

an iterative process. However, the introduction at each step of a certain substructure allows the formation of modules and results in a final graph with a self-similar structure. 


\subsection{Iterative algorithm to generate the graph $H(t)$}

The graph $H(t)$ is constructed as follows: For $t=0, H(0)$ is $C_{4}$, a length four cycle. We define now as generating cycle a cycle $C_{4}$ whose vertices have not been introduced at the same iteration step and passive cycle a cycle $C_{4}$ which does not verify this property. For $t \geq 1, H(t)$ is obtained from $H(t-1)$ by considering all their generating cycles $C_{4}$ and connecting, vertex to vertex, to each of them a new cycle $C_{4}$. This operation is equivalent to adding to the graph a cube $Q_{3}$ by identifying vertex to vertex the generating cycle with one of the cycles of $Q_{3}$. The process is repeated until the desired graph order is reached.
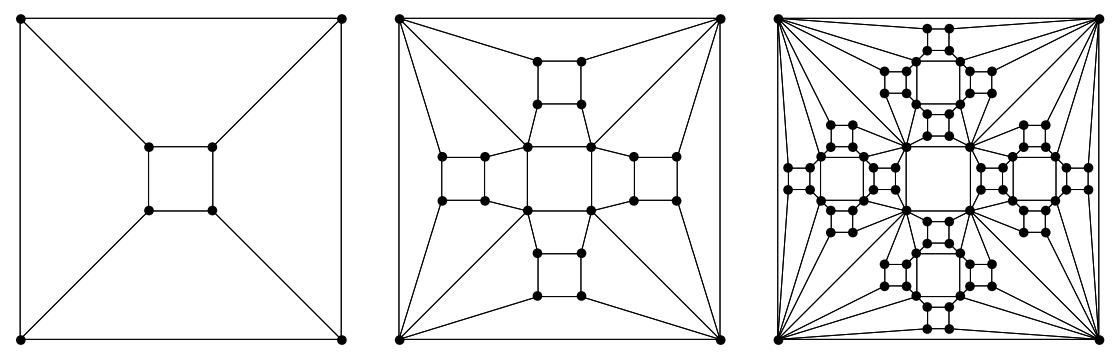

Figure 1. Graphs $H(t)$ produced at iterations $t=1,2$ and 3.

\subsection{Recursive modular construction}

The graph $H(t)$ can be also defined as follows: For $t=0, H(0)$ is the cycle $C_{4}$. For $t \geq 1, H(t)$ is produced from four copies of $H(t-1)$ by identifying, vertex to vertex, the initial passive cycle of each $H(t-1)$ with each of four consecutive cycles of $Q_{3}$ (leaving two opposite cycles of $Q_{3}$ free), see Fig. 2.
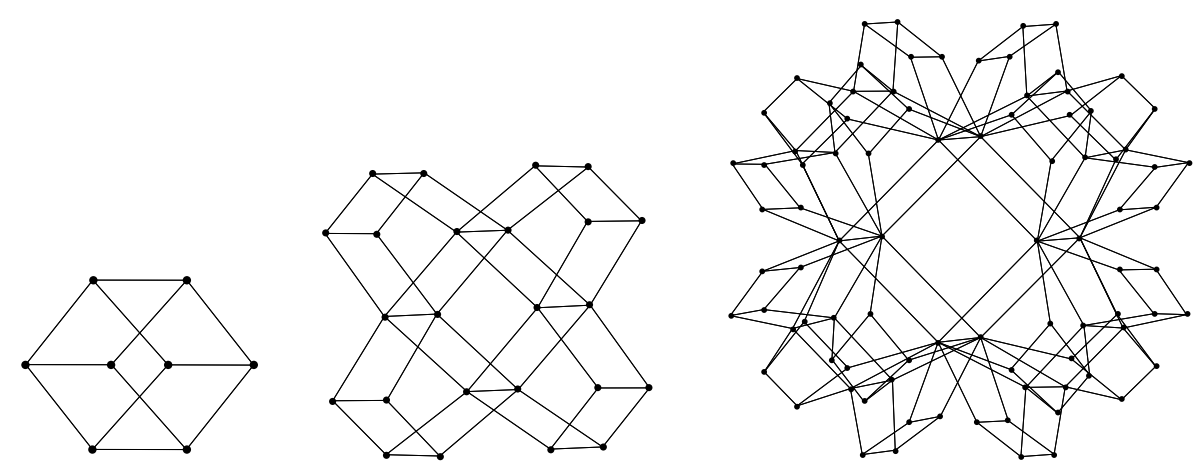

Figure 2. Modular construction of $H(t)$ for $t=1,2$ y 3 . At step $t$, we merge four copies of $H(t-1)$ to four cycles of the cube $Q_{3}$, leaving opposite cycles free. See the text for details. 


\begin{tabular}{cccc}
\hline Step & Vertices & Edges & Number of active cycles \\
\hline & & & \\
0 & 4 & 4 & 1 \\
1 & 8 & 12 & 4 \\
2 & 24 & 44 & 16 \\
3 & 88 & 172 & 64 \\
$\ldots$ & $\ldots$ & $\cdots$ & $\ldots$ \\
$\mathrm{t}$ & $\frac{4^{t+1}+8}{3}$ & $\frac{2 \cdot 4^{t+1}+4}{3}$ & $4^{t}$ \\
$\ldots$ & $\cdots$ & $\cdots$ & $\cdots$ \\
\hline
\end{tabular}

Table 1. Number of vertices, edges and generating cycles of $H(t)$ at each step.

\subsection{Properties of $H(t)$}

Order and size of $H(t)$. - We use the following notation: $\tilde{V}(t)$ and $\tilde{E}(t)$ denote, respectively, the set of vertices and edges introduced at the step $t$, while $V(t)$ and $E(t)$ denote the set of vertices and edges of the graph $H(t) . \tilde{C}(t)$ is the number of generating cycles $C_{4}$ at step $t$, which will be used to produce the graph $H(t+1)$.

Note that at each iteration, any generating cycle is replaced by four new generating cycles and one passive cycle. Therefore: $\tilde{C}(t+1)=4 \cdot \tilde{C}(t), t \geq 1$ and $\tilde{C}(0)=1$. Thus $\tilde{C}(t)=4^{t}$. Moreover, each generating cycle introduces at the next iteration four new vertices and eight new edges. As a consequence, $\tilde{V}(t)=4 \cdot \tilde{C}(t-1)=4 \cdot 4^{t-1}$ and $\tilde{E}(t)=8 \cdot \tilde{C}(t-1)=8 \cdot 4^{t-1}=2 \cdot 4^{t}$, thus:

$$
|V(t)|=\sum_{i=0}^{t} \tilde{V}(t)=\frac{4^{t+1}+8}{3} \quad|E(t)|=\sum_{i=0}^{t} \tilde{E}(t)=\frac{2 \cdot 4^{t+1}+4}{3}
$$

Degree distribution. - Intially, at $t=0$, the graph is a single generating cycle $C_{4}$ and its four vertices have degree two.

When a new vertex $i$ is added to the graph at iteration $t_{i}\left(t_{i} \geq 1\right)$, it has degree 3 . We denote by $C(i, t)$ the number of generating cycles at iteration $t$ which will produce new vertices that will connect to vertex $i$ at step $t+1$. At iteration $t_{i}$, when vertex $i$ is introduced, the value of $C\left(i, t_{i}\right)$ is 2 . According to the construction process of the graph, at each iteration, each new neighbor of $i$ belongs to two generating cycles where $i$ is also a vertex. If we denote as $k(i, t)$ the degree of vertex $i$ at step $t$, then we have the following relationship: $C(i, t)=k(i, t)-1$.

We now compute $C(i, t)$. As we have seen above, each generating cycle to which $i$ belongs, produces two new generating cycles which also have $i$ as a vertex. Thus $C(i, t)=2 \cdot C(i, t-1)$. Using the initial condition $C\left(i, t_{i}\right)=2$, we have $C(i, t)=2^{t-t_{i}+1}$. Therefore the degree of vertex $i$ at the step $t$ is

$$
k(i, t)=2^{t-t_{i}+1}+1 .
$$

Note that the initial four vertices of step 0 follow a different process. In this case $C(i, 0)=2^{t}$ and $k(i, t)=2^{t}+1$. Thus, at step $t$ the initial four vertices of the graph 


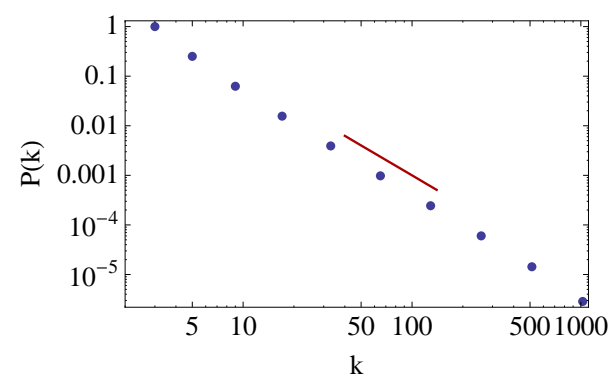

Figure 3. Log-log representation of the cumulative degree distribution for $H(10)$ with $|V|=1398104$ vertices. The reference line has slope -2 .

have the same degree than those introduced at step 1.

From equation (2) we verify that the graph has a discrete degree distribution and we use the technique described by Newman in [2] to find the cumulative degree distribution $P_{\text {cum }}(k)$ for a vertex with degree $k: P_{\text {cum }}(k)=\sum_{\tau \leq t_{i}}|V(\tau)| /\left|V\left(t_{i}\right)\right|=$ $\left(4^{t_{i}+1}+8\right) /\left(4^{t+1}+8\right)$.

Replacing $t_{i}$, from equation (2), in the former equation $t_{i}=t+1-\ln (k-1) / \ln 2$ we obtain $P_{\text {cum }}(k)=\left(16 \cdot 4^{t} \cdot(k-1)^{-2}+8\right) /\left(4^{t}+8\right)$, which for large values of $t$, allows us to write $P_{\text {cum }}(k) \sim k^{1-\gamma_{k}}=k^{-2}$, and therefore the degree distribution, for large graphs, follows a power-law with exponent $\gamma_{k}=3$. Research on networks associated to electronic circuits (these networks show planarity, modularity and a small clustering coefficient) gives similar values for their degree power-law distribution [22, 2]. More precisely, the largest benchmark considered -a network with 24097 nodes, 53248 edges, average degree 4.34 and average distance 11.05- has a degree distribution which follows a power-law with exponent 3.0, precisely the same as in our model, and it has a small clustering coefficient $C=0.01$.

Diameter.- At each step we introduce, for each generating cycle, four new vertices which will form a new cycle $C_{4}$ (and these vertices are among them at maximum distance 2). As all join the graph of the former step with one new edge, the diameter will increase by exactly 2 units. Therefore $D_{t}=D_{t-1}+2$. $t \geq 2$. As $D_{1}=3$, we have that the diameter of $H(t)$ is $D_{t}=2 \cdot t+1$ if $t \geq 1$. Therefore, from Eq.1, and as for $t$ large $t \sim \ln \left|V_{t}\right|$ we have in this limit that $D_{t} \propto \ln \left|V_{t}\right|$.

Average distance. - The average distance of $H(t)$ is defined as:

$$
\bar{d}_{t}=\frac{1}{|V(t)|(|V(t)|-1) / 2} \sum_{i, j \in V(t)} d_{i, j},
$$

where $d_{i, j}$ is the distance between vertices $i$ and $j$. We will denote as $S_{t}$ the sum $\sum_{i, j \in V(t)} d_{i, j}$.

The modular recursive construction of $H(t)$ allows us to calculate the exact value of $\bar{d}_{t}$. At step $t, H(t+1)$ is obtained from the juxtaposition of four copies of $H(t)$, which we label $H_{t}^{\varphi}, \varphi=1,2,3,4$, on top of the cube $Q_{3}$ (see Figs. 2 and 4). The copies are connected one to another at the vertices which we call connecting vertices and we label 


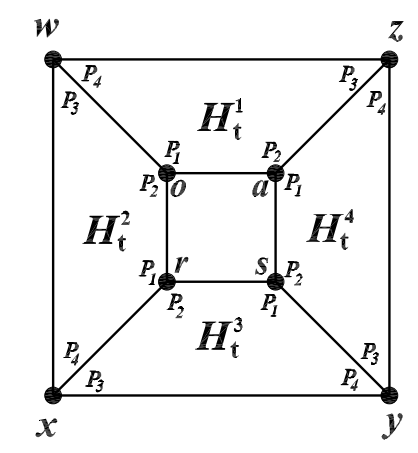

Figure 4. Classification of nodes of $H(t+1)$ into classes $H_{t}^{\varphi}, \varphi=1,2,3,4$.

$w, x, y, z, o, r, s$, and $a$. The other vertices of $H(t+1)$ will be called interior vertices. Thus, the sum of distances distance $S_{t+1}$ satisfies the following recursion:

$$
S_{t+1}=4 S_{t}+\Delta_{t}-4
$$

where $\Delta_{t}$ is the sum over all shortest paths whose endvertices are not in the same $H(t)$ copy and the last term compensates for the overcounting of the paths corresponding to $d_{w, o}, d_{x, r}, d_{s, y}$, and $d_{z, a}$-for example, $d(w, o)$ is included both in $H_{t}^{1}$ and $H_{t}^{2-}$. Note that the paths that contribute to $\Delta_{t}$ must all go through at least one of the eight connecting vertices.

The analytical expression for $\Delta_{t}$ is not difficult to find. We denote as $\Delta_{t}^{\alpha, \beta}$ the sum of all shortest paths with endvertices in $H_{t}^{\alpha}$ and $H_{t}^{\beta} . \Delta_{t}^{\alpha, \beta}$ excludes the paths such that either endvertex is a connecting vertex, i.e. a path which contributes to $\Delta_{t}^{\alpha, \beta}$ must not end at nodes $w, x, y, z, o, r, s$, or $a$. Then the total sum $\Delta_{t}$ is

$$
\begin{gathered}
\Delta_{t}=\Delta_{t}^{1,2}+\Delta_{t}^{1,3}+\Delta_{t}^{1,4}+\Delta_{t}^{2,3}+\Delta_{t}^{2,4}+\Delta_{t}^{3,4}+20 \\
+\sum_{\substack{i \in H_{t}^{3} \cup H_{t}^{4}, i \notin x, r, s, y, a, z}}\left(d_{w, i}+d_{o, i}\right)+\sum_{\substack{i \in H_{t}^{1} \cup H_{t}^{4}, i \notin w, o, a, z, s, y}}\left(d_{x, i}+d_{r, i}\right) \\
+\sum_{\substack{i \in H_{t}^{1} \cup H_{t}^{2}, i \notin x, r, o, w, a, z}}\left(d_{s, i}+d_{y, i}\right)+\sum_{\substack{i \in H_{t}^{2} \cup H_{t}^{3}, i \notin w, o, x, r, s, y}}\left(d_{a, i}+d_{z, i}\right),
\end{gathered}
$$

where the term 20 comes from the sum of $d_{w, s}, d_{w, y}, d_{o, s}, d_{o, y}, d_{x, a}, d_{x, z}, d_{r, a}$, and $d_{r, z}$, and the last four sums count shortest paths ending in a connecting vertex.

By symmetry, $\Delta_{t}^{1,2}=\Delta_{t}^{1,4}=\Delta_{t}^{2,3}=\Delta_{t}^{3,4}, \Delta_{t}^{1,3}=\Delta_{t}^{2,4}$, and $\sum_{i} d_{w, i}=\sum_{i} d_{o, i}=$ $\sum_{i} d_{x, i}=\sum_{i} d_{r, i}=\sum_{i} d_{s, i}=\sum_{i} d_{y, i}=\sum_{i} d_{a, i}=\sum_{i} d_{z, i}$, and

$$
\Delta_{t}=4 \Delta_{t}^{1,2}+2 \Delta_{t}^{1,3}+20+8 \sum_{\substack{i \in H_{t}^{3} \cup H_{t}^{4}, i \notin x, r, s, y, a, z}} d_{w, i} .
$$

To calculate $\Delta_{t}$, we classify all interior vertices of $H(t+1)$ into four different classes according to their distances to each of the four vertices $w, x, y$, and $z$. The classes are denoted, respectively, $P_{1}, P_{2}, P_{3}$, and $P_{4}$. Vertices $w, x, y$, and $z$ are not considered 
for this classification. This classification is represented in Fig. 4. By construction, for an arbitrary interior vertex $v$, there must exist one of the above mentioned vertices (say $w)$ satisfying $d_{v, w}<d_{v, x}, d_{v, w}<d_{v, y}$, and $d_{v, w}<d_{v, z}$. All the interior vertices nearest to $w$ (resp. $x, y$, and $z$ ) are assigned to class $P_{1}$ (resp. $P_{2}, P_{3}$, and $P_{4}$ ). The total number of vertices of $H_{t}$ that belong to the class $P_{\tau}(\tau=1,2,3,4)$ is denoted by $N_{t, P_{\tau}}$. Since the four vertices $w, x, y$, and $z$ play a symmetrical role, classes $P_{1}, P_{2}, P_{3}$, and $P_{4}$ are equivalent. Thus, $N_{t, P_{1}}=N_{t, P_{2}}=N_{t, P_{3}}=N_{t, P_{4}}$ which will be abbreviated to $N_{t}$ from now on. We have

$$
N_{t}=\frac{\left|V_{t}\right|-4}{4}=\frac{4^{t}-1}{3} .
$$

We denote by $L_{t+1, P_{1}}\left(L_{t+1, P_{2}}, L_{t+1, P_{3}}, L_{t+1, P_{4}}\right)$ the sum of distances between vertices $w(x, y, z)$ and all interior vertices $v \in P_{1}\left(P_{2}, P_{3}, P_{4}\right)$ of $H(t+1)$. Because of the symmetry, $L_{t+1, P_{1}}=L_{t+1, P_{2}}=L_{t+1, P_{3}}=L_{t+1, P_{4}}$ that will be written as $L_{t+1}$ for short. Taking into account the recursive method of constructing $H(t)$ we notice that the vertex classification follows also a recursion. For example classes $P_{1}$ and $P_{4}$ in $H_{t}^{1}$, classes $P_{2}$ and $P_{2}$ in $H_{t}^{2}$, and one shared edge node $o$ belong to class $P_{1}$ in $H(t+1)$, see Fig. 4 . Therefore we can write the following recursive formula for $L_{t+1}$ :

$$
L_{t+1}=4 L_{t}+2 N_{t}+1 \text {. }
$$

We can solve Eq. (8) inductively, with initial condition $L_{1}=1$, and we have

$$
L_{t}=\frac{1}{18}\left(3 t \cdot 4^{t}+2 \cdot 4^{t}-2\right) \text {. }
$$

We now return to compute Eq. (6), with $\Delta_{t}^{1,2}$ given by the sum

$$
\Delta_{t}^{1,2}=\sum_{\substack{u \in H_{t}^{1}, u \notin\{w, o, a, z\} ; \\ v \in H_{t}^{2}, v \notin\{w, x, r, o\}}} d_{u, v}=\sum_{i=1}^{4} \sum_{j=1}^{4} d_{P_{i}^{t, 1}, P_{j}^{t, 2}}
$$

where $P_{i}^{t, 1}$ and $P_{j}^{t, 2}$ are the vertex classes $P_{i}$ and $P_{j}$ of $H_{t}^{1}$ and $H_{t}^{2}$, respectively, and $d_{P_{i}^{t, 1}, P_{j}^{t, 2}}$ is the sum of distances $d_{u, v}$ for all vertices $u \in P_{i} \subset H_{t}^{1}$ and $v \in P_{j} \subset H_{t}^{2}$.

We have:

$$
d_{P_{1}^{t, 1}, P_{1}^{t, 2}}=\sum_{\substack{u \in P_{1} \subset H_{t}^{1}, v \in P_{1} \subset H_{t}^{2}}} d_{u, v}=\sum_{\substack{u \in P_{1} \subset H_{t}^{1} \\ v \in P_{1} \subset H_{t}^{2}}}\left(d_{u, o}+d_{o, r}+d_{r, v}\right)=2 N_{t} L_{t}+N_{t}^{2} .
$$

In a similar way, we can obtain the expressions $d_{P_{i}^{t, 1}, P_{j}^{t, 2}}$ for different values of $i$ and $j$ in Eq. (10). The results are $d_{P_{1}^{t, 1}, P_{2}^{t, 2}}=d_{P_{4}^{t, 1}, P_{3}^{t, 2}}=2 N_{t} L_{t}, d_{P_{1}^{t, 1}, P_{1}^{t, 2}}=d_{P_{1}^{t, 1}, P_{3}^{t, 2}}=$ $d_{P_{2}^{t, 1}, P_{2}^{t, 2}}=d_{P_{3}^{t, 1}, P_{3}^{t, 2}}=d_{P_{4}^{t, 1}, P_{2}^{t, 2}}=d_{P_{4}^{t, 1}, P_{4}^{t, 2}}=2 N_{t} L_{t}+\left(N_{t}\right)^{2}, d_{P_{1}^{t, 1}, P_{4}^{t, 2}}=d_{P_{2}^{t, 1}, P_{1}^{t, 2}}=$ $d_{P_{2}^{t, 1}, P_{3}^{t, 2}}=d_{P_{3}^{t, 1}, P_{2}^{t, 2}}=d_{P_{3}^{t, 1}, P_{4}^{t, 2}}=d_{P_{4}^{t, 1}, P_{1}^{t, 2}}=2 N_{t} L_{t}+2\left(N_{t}\right)^{2}$, and $d_{P_{2}^{t, 1}, P_{4}^{t, 2}}=d_{P_{3}^{t, 1}, P_{1}^{t, 2}}=$ $2 N_{t} L_{t}+3\left(N_{t}\right)^{2}$. Inserting these results into Eq. (10), we have

$$
\Delta_{t}^{1,2}=32 N_{t} L_{t}+24\left(N_{t}\right)^{2} .
$$

Analogously, we can obtain

$$
\Delta_{t}^{1,3}=32 N_{t} L_{t}+32\left(N_{t}\right)^{2} .
$$


Now, to find an expression for $\Delta_{t}$, the only thing left is to evaluate the last term of Eq. (6), which can be obtained as above

$$
\begin{aligned}
& 8 \sum_{\substack{i \in H_{t}^{3} \cup H_{t}^{4} ; \\
i \notin x, r, s, y, a, z}} d_{w, i}=16 \sum_{i \in H_{t}^{3}, i \notin x, r, s, y} d_{w, i}= \\
& 16 \sum_{\substack{i \in P_{1} \subset H_{t}^{3} \\
+16}}\left(d_{w, s}+d_{s, i}\right)+16 \sum_{i \in P_{2} \subset H_{t}^{3}}\left(d_{w, r}+d_{r, i}\right)+ \\
& =64 L_{t \in P_{2} \subset H_{t}^{3}}\left(d_{w, x}+d_{x, i}\right)+16 \sum_{i \in P_{4} \subset H_{t}^{3}}\left(d_{w, y}+d_{y, i}\right)= \\
&
\end{aligned}
$$

Substituting Eqs. (12), (13) and (14) into Eqs. (6) and (4), we obtain the recursive expression for the total distance $S_{t}$ :

$$
S_{t+1}=4 S_{t}+192 N_{t} L_{t}+160\left(N_{t}\right)^{2}+64 L_{t}+128 N_{t}+16 .
$$

Substituting Eq. (7) for $N_{t}$ and Eq. (9) for $L_{t}$ into Eq. (15), and using $S_{0}=8$, we have

$$
S_{t+1}=\frac{8}{27}\left[10+14 \cdot 4^{t}+3(t+1) \cdot 16^{t}\right] .
$$

Inserting Eq. (16) into Eq. (3), the analytical expression for $\bar{d}_{t}$ can be obtained as

$$
\bar{d}_{t}=\frac{4}{3} \cdot \frac{10+14 \cdot 4^{t}+3(t+1) 16^{t}}{10+13 \cdot 4^{t}+4 \cdot 16^{t}} .
$$

Notice that for a large order $(t \rightarrow \infty) \bar{d}_{t} \simeq t+1 \sim \ln \left|V_{t}\right|$, which means that the average distance shows a logarithmic scaling with the order of the graph, and has a similar behavior as the diameter (the graph is small-world).

Strength distribution. - The strength of a node in a network is associated to resources or properties allocated to it, as the total number of publication of an author, in the case of the network associated to the Erdös number; the total number of passengers in the world-wide airports network, etc.

In our case we associate to each vertex the area of the passive cycle, defined by the four vertices introduced at a given step. For this purpose we assume a uniform construction of the graph. At the initial step the area is $\mathcal{A}_{0}$ and we denote as $\mathcal{A}_{t}$ the area of the passive cycle introduced at step $t$. By convention, we establish that the area of this cycle is one fifth of the area of the cycle where it connects (as each introduction of a passive cycle is associated to the simultaneous introduction of four generating cycles). Therefore we have $\mathcal{A}_{t}=\left(\frac{1}{5}\right)^{t} \mathcal{A}_{0}$. A vertex $i$ introduced at $t_{i}$ will have strength $s\left(i, t_{i}\right)=\left(\frac{1}{5}\right)^{t_{i}} \mathcal{A}_{0}$ and it will keep it i n further steps $t>t_{i}$. As we want to find the strength distribution for all vertices of the graph at step $t$, we have that $s\left(i, t_{i}\right)=\left(\frac{1}{5}\right)^{t_{i}-t} \cdot \mathcal{A}_{t}$.

Using equation (2) we obtain the following power-law for the correlation between the strength and the degree of a vertex:

$$
s(i, t)=\frac{1}{5} \mathcal{A}_{t}(k(i, t)-1)^{\ln 5 / \ln 3},
$$


which for large values of the degree $k$ leads to $s(k) \sim k^{\ln 5 / \ln 3}$.

We should mention that similar exponents have been found for the relation between the strength and the degree of the node of real life networks like the airports network, Internet and the scientist collaboration graph [23].

After a similar analysis to the calculation of the degree distribution, we find that the strength distribution also follows a power law with exponent:

$$
\gamma_{s}=1+2 \frac{\ln 2}{\ln 5} .
$$

It has been shown that if a weighted graph with a non-linear correlation between strength and degree $s(k) \sim k^{\beta}$ and the degree and strength distributions follows power laws, $P(k) \sim k^{-\gamma_{k}}$ and $P(s) \sim s^{-\gamma_{s}}$, then there exists a general relationship between $\gamma_{k}$ and $\gamma_{s}$ given by $\gamma_{s}=\frac{\gamma_{k}}{\beta}+\frac{\beta-1}{\beta}$ [23].

From the former relationship, and as we have $\gamma_{k}=3$ and $\beta=\ln 5 / \ln 3$, the exponent

of the strength distribution is $\gamma_{s}=3 \frac{\ln 2}{\ln 3}+\ln 2\left(\frac{\ln 5}{\ln 2}-1\right) / \ln 5$, and we obtain the same value $\gamma_{s}$ (19) which was computed directly.

\section{Conclusion}

The family of graphs introduced and studied here has as main characteristics planarity, modularity, degree hierarchy, and small-world and scale-free properties. At the same time the graphs have clustering zero. A combination of modularity and scale-free properties is present in many real networks like those associated to living organism (protein-protein interaction networks) and some social and technical networks [18, 20]. The added property of a small clustering coefficient appears also in some technological networks (electronic circuits, Internet, P2P) and social networks [2, 22]. Therefore our model, with a null clustering coefficient, could be considered to model these networks and also it can be used to study other properties without the influence of the clustering. The deterministic character of the family, as opposed to usual probabilistic models, should facilitate the exact computation of many network parameters.

On the other hand, simple variations of our model allow the introduction of clustering. As an example, by adding to each passive cycle an edge we can introduce two triangles for each cycle and therefore obtain a planar graph with non-zero clustering. Replacing in the construction each passive cycle by a complete graph $K_{4}$ will produce a family with a relatively large clustering coefficient. However the graph will no longer be planar.

\section{Acknowledgments}

Research supported by the Ministerio de Educación y Ciencia, Spain, and the European Regional Development Fund under project TEC2005-03575 and by the Catalan Research Council under project 2005SGR00256. L. Chen and Z. Zhang are supported by the National Natural Science Foundation of China under Grant No. 60704044, the 
Postdoctoral Science Foundation of China under Grant No. 20060400162, and the Huawei Foundation of Science and Technology (YJCB2007031IN).

\section{References}

[1] Albert R and Barabási A-L 2002 Statistical mechanics of complex networks Rev. Mod. Phys. 74 $47-97$

[2] Newman M E J 2003 The structure and function of complex networks SIAM Review ,45 167-256

[3] Song C, Havlin S and Makse H A 2005 Self-similarity of complex networks. Nature, 433 392-395

[4] Song C, Havlin S and Makse H A 2006 Origins of fractality in the growth of complex networks Nature Phys. 2 275-281

[5] Goh K I, Salvi G, Kahng B and Kim D 2006 Skeleton and fractal scaling in complex networks Phys. Rev. Lett. 96018701

[6] Kim J S, Goh K I, Kahng B and Kim D 2007 Fractality and self-similarity in scale-free networks New J. Phys. 9177

[7] Beineke L W and Pippert R E 1971 Properties and characterization of k-trees Mathematika 18 $141-151$

[8] Dorogovtsev S N, Goltsev A V and Mendes J F F 2002 Pseudofractal scale-free web Phys. Rev. E 65066122

[9] Jung S, Kim S, and Kahng B 2002 Geometric fractal growth model for scale-free networks Phys. Rev. E 65056101

[10] Comellas F, Fertin G and Raspaud A 2004 Recursive graphs with small-world scale-free properties Phys. Rev. E 69037104

[11] Andrade J S Jr., Herrmann H J, Andrade R F S, and da Silva L R 2005 Apollonian Networks: Simultaneously scale-free, small world, Euclidean, space filling and with matching graphs Phys. Rev. Lett. 94018702

[12] Doye J P K and Massen C P 2005 Self-similar disk packings as model spatial scale-free networks Phys. Rev. E 71016128

[13] Zhang Z Z, Comellas F, Fertin G and Rong L L 2006 High dimensional Apollonian networks $J$ Phys A: Math Gen 39 1811-1818

[14] Chung F, Lu L, Dewey T G and Galas D J 2003 Duplication models for biological networks J. Comput. Biol. 10 677-687,

[15] Zhang Z Z, Zhou S, Fang L, Guan J and Zhang Y 2007 Maximal planar scale-free Sierpinski networks with small-world effect and power law strength-degree correlation. Europhys. Lett. 79 38007

[16] Barabási A-L and Oltvai Z N 2004 Network biology: Understanding the cell's functional organization Nature Rev. Genetics 5 101-113

[17] Noh J D 2003 Exact scaling properties of a hierarchical network model Phys. Rev. E 67045103

[18] Ravasz E, Barabási A-L. 2003 Hierarchical organization in complex networks Phys. Rev. E 67 026112

[19] Jeong H, Tombor B, Albert R, Oltvai Z N and Barabási A-L 2000 The large-scale organization of metabolic networks Nature 407 651-654

[20] Ravasz E, Somera A L, Mongru D A, Oltvai Z N and Barabási A-L 2002 Hierarchical organization of modularity in metabolic networks Science 297 1551-1555

[21] Barriere L, Comellas F, Dalfó C and Fiol M A 2008 The hierarchical product of graphs Discrete Appled Math. doi:10.1016/j.dam.2008.04.018

[22] Ferrer i Cancho R, Janssen C and Solé R V 2001 Topology of technology graphs: Small world patterns in electronic circuits Phys. Rev. E 64046119

[23] Barrat A, Barthélemy M, Pastor-Satorras R and Vespignani A 2004 The architecture of complex weighted networks Proc. Natl. Acad. Sci. U.S.A. 101 3747-3752 